This is a self-archived version of an original article. This version may differ from the original in pagination and typographic details.

Author(s): Välisalo, Tanja; Ruotsalainen, Maria

Title: "I never gave up" : engagement with playable characters and esports players of Overwatch

Year: 2019

Version: Accepted version (Final draft)

Copyright: (c) 2019 ACM

Rights: In Copyright

Rights url: http://rightsstatements.org/page/InC/1.0/?language=en

Please cite the original version:

Välisalo, T., \& Ruotsalainen, M. (2019). "I never gave up" : engagement with playable characters and esports players of Overwatch. In FDG '19: Proceedings of the International Conference on the Foundations of Digital Games (Article 40). ACM. https://doi.org/10.1145/3337722.3337769 


\section{"I Never Gave Up"}

\section{Engagement with Playable Characters and Esports Players of Overwatch}

\author{
Tanja Välisalo \\ Department of Music, Art and Culture Studies \\ University of Jyvaskyla \\ Jyvaskyla, Finland \\ tanja.valisalo@jyu.fi
}

\author{
Maria Ruotsalainen \\ Department of Music, Art and Culture Studies \\ University of Jyvaskyla \\ Jyvaskyla, Finland \\ maria.a.t.ruotsalainen@jyu.fi
}

\begin{abstract}
Esports phenomena have grown rapidly in recent years, and so has research on the topic. Some of the research has also addressed esports fandom (see e.g. Taylor 2012). Nevertheless, studies comparing and contrasting how players and fans engage with the game and the esports based on that game are scarce. This study compares and contrasts how players and fans engage with playable game characters and esports players. The paper draws on previous research in fan studies, sports fandom and esports to examine the relationships of players and fans of the videogame Overwatch (Blizzard Entertainment 2016) with the fictional heroes of the game as well as with their favorite professional players in the newly started Overwatch League. We analyse how these relationships are articulated by fans and players, and pay attention to emerging similarities and differences.
\end{abstract}

The findings show that personality is deemed important for engagement with both, game characters and esports players. In addition, gender and sexual orientation emerged as important factors. By contrast, nationality was deemed important for engagement with esports players, but not with player characters. Further research should concentrate on the connections between esports and identity politics, as well as player characters and identity construction.

https://doi.org/10.1145/3337722.3337769

\section{CCS CONCEPTS}

- Information systems Massively multiplayer online games - Social and professional topics $\sim$ Gender $\bullet$ Social and professional topics $\sim$ Sexual orientation

\section{KEYWORDS}

Game Characters, Esports, Esports Players, Fans, Overwatch

Permission to make digital or hard copies of all or part of this work for personal or classroom use is granted without fee provided that copies are not made or distributed for profit or commercial advantage and that copies bear this notice and the full citation on the first page. Copyrights for components of this work owned by others than the author(s) must be honored. Abstracting with credit is permitted. To copy otherwise, or republish, to post on servers or to redistribute to lists, requires prior specific permission and/or a fee. Request permissions from Permissions@acm.org. FDG '19, August 26-30, 2019, San Luis Obispo, CA, USA

(C) 2019 Copyright is held by the owner/author(s). Publication rights licensed to ACM.

\section{ACM Reference format:}

Tanja Välisalo and Maria Ruotsalainen. 2019. "I Never Gave Up”* Engagement with Playable Characters and Esports Players of Overwatch. In Proceedings of FDG '19, August 26-30, 2019, San Luis Obispo, CA, USA, 6 pages. https://doi.org/10.1145/3337722.3337769

\section{Introduction}

Esports have been on the rise in the past years and have also caught the attention of the academia. Esports have, for example, been studied as a form of media sports as well as a growing new business, and additionally, have been used as an opportunity to study high-achieving players. Some of the research has also addressed esports fandom (see e.g. Taylor 2012). Nevertheless, studies examining the players' and fans' relationship to both a videogame and its fictional world as well as esports based on that particular videogame are scarce. Our research addresses this gap by examining the relationships of players and fans of the videogame Overwatch (Blizzard Entertainment 2016) with the fictional heroes of the game as well as with their favorite professional players in the newly started Overwatch League. In essence, we are examining the relationship between game fandom and esports fandom.

The two types of fandom have traditionally been researched separately. Fandom studies has been concerned with fans of fiction and music, while sports fandom has been a somewhat separate area of interest. Our study approaches these two phenomena with similar data and methods. We collected discussion forum data, and conducted a survey in order to analyze the ways fans discuss their favorite game characters and professional players. This data allows us to examine, how fans and players articulate these relationships, and identify the emerging similarities and differences.

The analysis of these relationships is informed by theories of audience engagement with fictional characters (e.g. Phelan 1989; Phelan, Rabinowitz, Warhol et al. 2012; Smith 1995; Smith 2011) as well as fan studies (e.g. Booth 2010) and the study of sports fandom (e.g. Crawford 2004). The results of this study will contribute to the emerging field of study on game fandom in addition to esports research.

\subsection{Overwatch and Overwatch League}

Team-based online multiplayer first-person shooter Overwatch was published in 2016 by Blizzard Entertainment. By 2017 it had 
gathered over 35 million active accounts (Activision Blizzard 2018), won game of the year award and 2018 it won the esport game of the year award. Placed somewhere in the distant future, Overwatch brings us the heroes of a former squad known as the "Overwatch". The squad's task was to ensure peace and protect people across the globe from the robotic "omnics", as well the enemies of this former squad and its allies. All of them - former members of Overwatch, its enemies and omnics - together form the diverse pool of playable heroes: from an omnic (robotic) monk to genius gorilla raised in space Overwatch offers a large variety of different heroes to be played. This is also visible in the gender division of the heroes as there is a fairly equal balance between male and female heroes - while the robotic omnics remain a minority. Different sexual orientations are also present, albeit these only come visible in the comics related to game - Overwatch is designed as a transmedial product, and its story unveils mainly on the comics and shorts animations related to the game, not in the game itself. Furthermore, the game presents a number of different ethnicities and nationalities as part of its character roster. Blizzard has also stated that Overwatch was designed to be "inclusive", so the design choices have been conscious ones.

Overwatch is described as team-based first person shooter, but its playable heroes can also perform multiple other kinds of abilities than just shooting: depending on the hero, one can, for example, heal or damage through auto-aim beam and for instance "boop", i.e. push enemies off the map with, certain abilities. Similarly to the diverse hero design, the multiplicity of different abilities can be seen as attractive to a diverse player-base, even to those who do not have previous experience in playing shooter games. Thus, regardless of being a first-person shooter (FPS) game, it provides opportunities also for players not skilled in aiming. Indeed, the game has gathered many "casual" players and players new to FPS.

Since the open beta, Overwatch has had an active competitive scene, but it was in early 2018 that Overwatch as an esport gained momentum with the launch of the Overwatch League (OWL). OWL is a global esports league, which had 12 teams in its inaugural season (20 as of now). What sets OWL apart from many other esports leagues is that, following traditional sports, the teams represent different cities rather than established esports organizations. While the league is international, majority of the teams represent cities in USA. OWL is a franchised league, in which it furthermore follows traditional (USA based) team sport leagues. OWL is also fully owned by Blizzard, the owner and developer of Overwatch.

\section{Theoretical background}

While there are many overlaps between game cultures and fan cultures, there is still relatively little research bridging this gap between fields. While game studies has often foregrounded interactivity between players and game, interactivity is also emphasized in the study of fans (e.g. Booth 2010). Fan studies has for long demanded for fans' self-identification in order to call them fans. Even though not all players or esports spectators identify as fans (e.g. Wirman 2007), the lens of fan studies can be applied to research of game communities. In recent years fan studies approaches have also been applied to communities and phenomena traditionally outside its scope, such as audiences of classical music. Definitions of fandom have also been updated. For example, Cornel Sandvoss defines fandom as a regular, emotionally involved consumption of a given popular text (Sandvoss 2005). Simultaneously, there has been a call for fandom studies approaches also from inside game studies. T.L. Taylor $(2012,188)$ has emphasized the importance of studying fandom for understanding gamers and game culture, e.g. play experience and identity formation.

Sports research is another important theoretical background for this study. Overwatch as an esport is marked by sportification, the process of reaching the status of sports (cf. Mora and Héas 2003). Sportification can also refer to a way elements from traditional sports are implemented as part of the representation of esports (Turtiainen et al. 2018). Furthermore, elements from traditional sports have been used as a way to legitimize esports and construct esports as a hypermasculine activity (Taylor, Jenson and de Castell 2009). Our data suggests that both media and fans continuously draw parallels between esports and sports and 52\% of our survey respondents indicated that they see esports as a "sport amongst other sports". Thus, it makes sense to take into account theories from the study of sport fans and fandom, particularly when examining how favorite players are discussed. This is all the more fruitful as research on sport fans is often quite separate from the study of popular culture fandom.

In fandom studies, the affective relationships of fans with certain cultural markers as well as the hierarchy formed by the structure and intensity of these relationships within the fandom are understood as building blocks of a constructed, performative and ever-changing identity (Grossberg 1992, 58-59; Booth 2010, 20, $60)$. Fans continuously observe and construct parallels between themselves and the object of their fandom thus making fan objects extensions of themselves, functioning simultaneously apart from and part of themselves (Sandvoss 2005, 96-97, 102-103). The constructed nature of this process shows for example in research on British football fans, which shows how fans of the same team can build differing, even contradictory meanings, of the same team, as "winners" or "losers" based on their individual self-reflective connection to the fandom (Sandvoss 2005, 105).

According to Crawford (2004), being a sport fan is often tied to locality and nationality - fans are often fans of the local team or/and the national team. Sports fandom is also perceived as a predominantly masculine activity and women can have hard time negotiating their positions in often quite hierarchical sports fandom (Crawford 2004). According to Taylor, Jenson and de Castell (2009) women face similar difficulties while participating in esports and the world of esports is often described by terms such as neoliberal masculinity (Voorhees 2015), hegemonic masculinity (Taylor 2012), and toxic meritocracy (Paul 2018), referring to the obstacles women and minorities face when trying to enter to the world of esports and the norm of the esport player as white or Asian young male. 


\section{Data and Methods}

For our analysis, we have utilized two different datasets. Firstly, we have used data gathered from three different discussions forums: The formal official Overwatch forums (the United Stated version) maintained by Blizzard (closed in February 2018); the current official Overwatch forums (the United Stated version) maintained by Blizzard and subreddit (i.e. category) r/competitiveoverwatch on the Reddit discussion forum. Secondly, we have utilized survey data with 135 respondents gathered in August and September 2018.

This paper and its focus is a part of larger, ongoing research on Overwatch and Overwatch League as well different practices, communities and cultures surrounding them. As so, the survey questionnaire was designed based on the preliminary analysis of parts of the forum data. Designing the questionnaire also drew from earlier research on character engagement (Välisalo 2017) as well as authors' experience working with massive reception study projects: the Hobbit research project and the Game of Thrones research project.

The forum data has been gathered in a number of ways. First, the data from old Blizzard Overwatch forum was gathered from the former official Overwatch discussions forums maintained by Blizzard Entertainment, the developer of Overwatch. The forum was closed in February 20, 2018, when the new forums were introduced. From the old forums, we gathered the discussion threads from the forum section "General" with number of search words. The data was gathered in February 2018. The earliest posts are from May 2016 and the latest from February 2018. This data mainly helped us to design the questionnaire. Second, another search was conducted in the current Blizzard Overwatch forums with search word "favorite", "favourite", "fan" and "fandom" but only discussion threads, which clearly discussed about favorite heroes or hero fandom were gathered. This search targeted posts from March 1, 2018 to August 1, 2018. The third forum data is gathered from the subreddit r/competativeoverwatch, which mainly hosts discussions about the Overwatch League. The search targeted all the posts up to 1.8.2018. Search words used were "favorite", "favourite", "fan" and "fandom", but only discussion threads, which clearly discussed about favorite players or favorite teams were gathered. The selection of search words was based on daily reading of all the forums since the beginning of 2018 .

In addition to the forum discussions, we analyzed data from survey. The survey data was gathered in August and September 2018 through opportunistic recruitment. Both those who play or/and watch Overwatch League were invited to answer. From the 135 respondents $42 \%$ identified as female, $56 \%$ as male, and $2 \%$ as other. Respondents were from multiple nationalities, but the majority were Finnish (48\%) and the second largest group were Americans (24\%). All of our respondents had played Overwatch and 112 respondents $(83 \%)$ had watched OWL.

As our method of analysis, we utilized close reading and thematic analysis (Guest, Macqueen and Namey 2012) on the forum data and applicable sections of the survey. For the forum data, we used data-driven coding using Atlas.ti software.

As our method of analysis, we utilized close reading and thematic analysis (Guest, Macqueen and Namey 2012) on the forum data and applicable sections of the survey. For the forum data, we used data-driven coding using Atlas.ti software.

\section{Character Fandom}

At the time of the survey, Overwatch had 28 playable heroes. Almost all respondents, $98.5 \%$, chose a favorite gameplay-based hero, and $91.0 \%$ chose a favorite lore-based hero. While both percentages are high, it is notable that clearly fewer respondents mention a favorite lore-based hero than a favorite gameplay-based hero. The choices were dispersed across different heroes with only two, Torbjörn and Wrecking Ball, not chosen by anyone in either question. The latter can be explained by Wrecking Ball being a new hero at the time, launched in July 2018.

There seems to be a gendered difference in which favorite characters were chosen. Reinhardt, a hypermasculine armored character in tank role, was the most popular lore-based hero in our survey, but barely any female respondents chose him. Male respondents chose Reinhardt most often as their favorite lore-based as well as gameplay-based character. Male respondents were far more likely to not have a favorite lore-based hero than female respondents.

Mercy was the most popular gameplay-based hero among the respondents identifying as female, but there was no clear preference amongst females in regards to a favorite lore-based hero. Even though there is a common perception that men tend to have more experience with aiming as they on average have played shooter games more and longer, it is notable that the player does not need to aim to play either Reinhardt or Mercy.

Respondents were asked why they chose a particular character as a favorite, and instructed to choose all the relevant options. The options for lore-based and gameplay-based characters were almost the same, with altogether ten options in common, with additional two options available for lore-based favorite heroes.

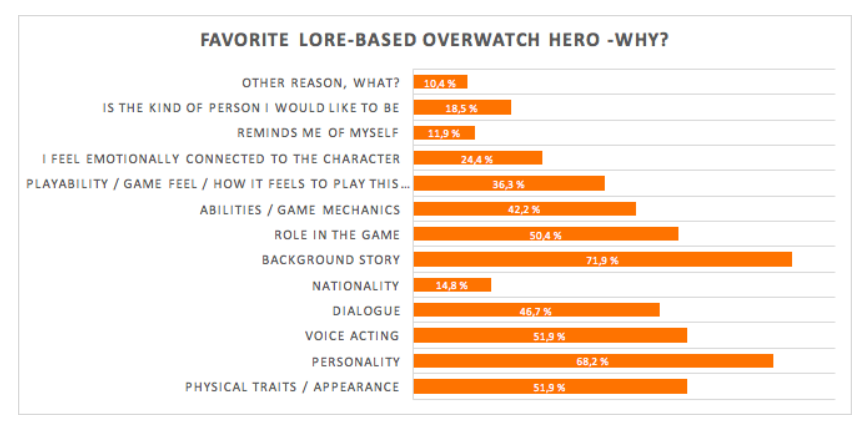

Figure 1: Reasons for choosing a favorite lore-based Overwatch hero. 


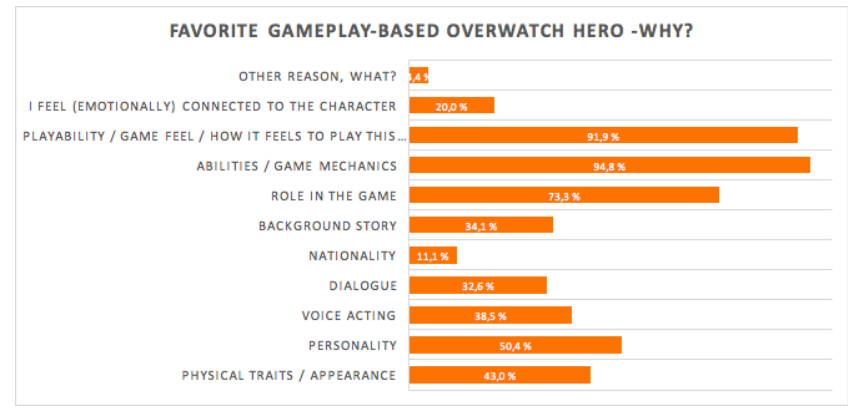

Figure 2: Reasons for choosing a favorite gameplay-based Overwatch hero.

Even though all available reasons for choosing a favorite hero appeared in responses to both questions, the prevalence of particular options differed quite expectedly between them: almost all respondents $(94.8 \%)$ chose "abilities / game mechanics" as a reason for their gameplay-based favorite, and most $(91.9 \%)$ mentioned "playability", while a large majority (73.3\%) also chose "role in the game" (see Figure 2). Respondents also had the opportunity to mention other reasons in their own words. Most common reasons here for game-play-based heroes were also related to their abilities, for example a particular mechanic, such as rocket punch for Doomfist. Most common reasons for choosing lore-based favorites (see Figure 1) were "background story" (71.9\%) and "personality" (68.2\%).

There were two additional options for lore-based favorites chosen by some respondents: "reminds me of myself" (11.9\%) and "is the kind of person I would like to be" (18.5\%) (see Figure 1). The open text answers provide additional descriptions for these choices, and include mentions of gender and sexual orientation. Forum discussions also reveal further personal nuances of character engagement: values, such as Mercy's pacifism and Doomfist's belief in war as a vehicle of positive change, are discussed as reasons for liking or disliking characters. This phenomenon is in previous studies described as allegiance to a fictional character (Smith 1995, 96).

Character abilities, manifested as mechanics, are not always distinguishable from character's personality and appearance in discussions on favorite or least favorite characters on the official Overwatch forum discussions. This provides background for interpreting survey responses and understanding why, for example, one in three respondents mention playability, which can be considered an element outside the narrative world, as a reason for choosing a lore-based favorite hero. Analysis of individual survey responses also shows that some options were chosen particularly often by the same respondents for both, lore-based and gameplaybased favorites: "physical traits / appearance", "personality", "voice acting" and "role in the game". This indicates that the socalled mimetic elements of a character (Phelan et al. 2012, 113), elements that make a character human-like, were important for engagement with a game character, whether the character was deemed important for its narrative or gameplay features.
Alterations to game characters are meaningful for players, which is evident in how character alterations gave rise to emotional accounts on the discussion forum. Discussions did not express only resistance to change, even though changes to the playable hero Mercy were discussed widely, but also contentment or desire for change: "I never gave up. When Reaper was trash, I never gave up. I always kept fighting for a buff. And what kept me going was not meaningless trust in someone else, or worthless love. It was faith in myself, and in Reaper." These affective articulations further demonstrate how character mechanics are interwoven with the player's emotional response to the character and the game.

\section{Player Fandom}

For the preparation of the survey and designing the questions, we analyzed discussions from Reddit's subreddit Competitive Overwatch, in order to see how favorite players are discussed and what kinds of reasons discussants gave for someone being their favorite player. From the discussions, nine different reasons emerged: the team the player is in, the history of the player (many of the OWL players have experience in competitive Overwatch preOWL, having played in pre-OWL teams and tournaments), a personal connection to the player, the place the player is from, player's gameplay, the hero the player plays, the player's personality, the player's stream, and knowing the player from other games. Based on these, we designed the survey questions to further explore the reasons for choices of favorite player.

In the survey, we had two questions related to favorite player: Firstly, we asked the respondents to name their favorite player. Here the Finnish players were highly presented, most likely due to large amount of Finnish respondents. This also indicates that nationality is a factor when choosing favorite player. Secondly, we asked with multiple-choice question "Why is this player your favorite?"

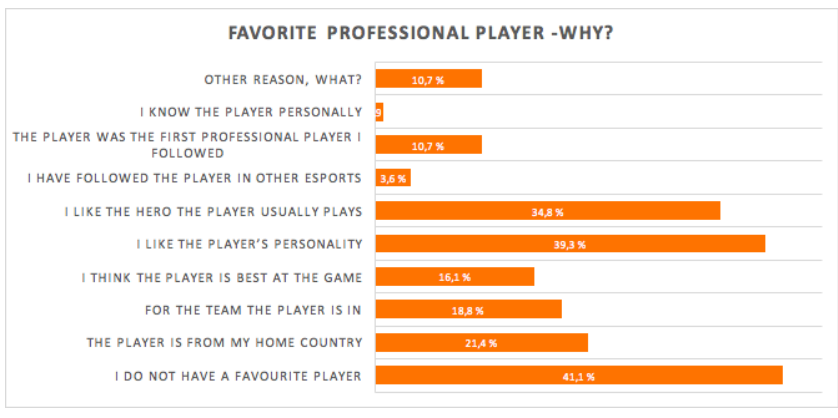

Figure 3: Reasons for choosing a favorite professional Overwatch player.

Altogether 113 of our 135 survey respondents indicated that they watch or have watched Overwatch League. Out of those, 59\% chose a favorite player. All of the respondents who indicated that they watch all the OWL games also chose a favorite player. The three most common reasons for "why is this player your favorite" chosen from given options were players personality (39\%), the hero the player plays (35\%) and the nationality of the player (21\%) (see 
Figure 3). The respondents were encouraged to choose as many reasons as they see fit.

When examining the reasons given for option "other reasons, what?" the gender of the player was given as a prominent reason: number of respondents indicated that Geguri, the only female player in OWL, was their favorite player and often singled out her gender as one of the reasons for that. One respondent wrote, "She is brave to be a woman in exports and rise above all the bullshit that female gamers go through. She is awesome for representation and shows other girls they can be pro gamers too". Another respondent articulated: "First female player in Overwatch, and I respect that greatly".

Another reason to arise from the "other reasons" was player's sexual orientation. In September 2018, when the responses for the survey were gathered, one player in OWL was openly gay. One response describes the meaning of this for the fan's own identity: "Because he's gay like me. (I'm not joking)".

\section{Comparing Fandoms}

When examining the reasons for favorite character and favorite players, there are overlapping factors: In both cases, gender as well as sexual orientation are deemed important - this came up in the open-ended questions. Personality, that of the favorite hero or that of the favorite player, was also deemed very important in both categories. Another major reason for someone being a favorite player was also the hero they played.

Clear differences emerged as well. While Overwatch heroes represent different nationalities - for instance, Reinhardt is German, and Mercy is from Switzerland - the nationality of the favorite hero does not appear to play a significant part in the ways fans choose their favorite hero. By contrast, nationality emerged as an important reason for choosing a favorite player. These results highlight the importance of bringing together the different research traditions in fandom - that of popular culture and that of sports - in order to understand the complex relationships fans forge with their favorite heroes and players. In the same vein, it needs to be asked why race nor ethnicity did not emerge as significant factors, even though previous literature suggests otherwise (i.e. Fletcher 2010; Young 2014). Overwatch also has a diverse cast of heroes from different ethnicities and races, while Overwatch League players are mainly white and Asian. It is worthwhile to note that this result might be influenced by the limited sample of this survey.

Furthermore, the survey indicated a difference between engagement with fictional characters in games and other media. Few respondents chose options related to identification with the game characters ("reminds me of myself" and "is the kind of person I would like to be"). This clearly differs from a previous study on film characters, where for the audiences of The Hobbit fantasy film trilogy the most common reason for choosing a favorite character was empathy or sympathy for the character (Välisalo 2017), most often expressed as finding the character "relatable" or "identifying" with the character. While similar reasons were present in the Overwatch survey data, they were chosen considerably less often.

\section{Conclusions}

In our paper, we presented results from our study on how players and fans of Overwatch and Overwatch League engage with playable heroes and esports players respectively. We analyzed how they articulate the reasons for their choices of favorite heroes and players to determine if there are similarities in their modes of engagement. We started the research by doing preliminary analysis of forum discussions and further implemented a survey.

We discovered that there are some significant overlaps, but also differences. Gender and sexual orientation emerged as important factors for engagement with both, the playable characters and esports players. We also found implications that there are differences in engagement with playable characters depending on the player's own gender identification. By contrast, nationality was important for engaging with esports players, but not with playable characters. These results show that some identity markers are more important for engagement with fictional characters than engagement with esports players thus demonstrating the diversity of engagements with the fan object in the culture surrounding Overwatch.

We found that mechanical and narrative elements of characters are intertwined in players' engagement with them. Further research combining traditional game studies approaches with study of media audiences and fandom studies is needed to delve deeper into the differences and similarities in engagement with fictional characters in different media. This will enrich the understanding of player's relationship with player characters and avatars.

Given how important nationality of the players is for Overwatch esports fans, it is noteworthy that the nationality of the playable heroes does not hold the same importance. It must be asked, if this is because characters' nationalities reside in the fictional world of the game where other narrative elements take precedence, or if the sportification process of esports emphasizes the importance of players' nationalities in esports fandom, similarly to traditional sports fandom.

Further research should concentrate on the connections between esports and identity politics, as well as player characters and identity construction. For instance, the significance of gender as well as sexual orientation needs to be further researched by building on existing literature. Examining the connection of these forms of engagement to player mentalities might also prove fruitful.

\section{ACKNOWLEDGMENTS}

This research was supported by the Academy of Finland project Centre of Excellence in Game Culture Studies (CoE-GameCult, 312397).

\section{REFERENCES}

[1] Activision Blizzard (2017, November 2). Activision Blizzard announces betterthan-expected third quarter 2017 financial results. Investor.activision.com.

[2] Paul Booth. 2010. Digital Fandom. New Media Studies. Peter Lang, New York.

[3] Garry Crawford. 2004. Consuming Sport. Fans, Sport and Culture. New York: Routledge.

[4] M Fletcher (2010). 'You must support Chiefs; Pirates already have two white fans!': race and racial discourse in South African football fandom, Soccer \& Society, 11(1-2), 79-94. 
[5] Allen Guttmann. 1978. From ritual to record. The nature of modern sports. Columbia University Press, New York.

[6] Greg Guest, Kathleen M. MacQueen and Emily E. Namey. 2012. Applied thematic analysis. Sage Publications, Los Angeles, California \& London.

[7] L Grossberg 1992 Is There a Fan in the House?: The Affective Sensibility of Fandom, The Adoring Audience: Fan Culture and Popular Media. Routledge. London and New York.

[8] VM Karhulahti (2017). Reconsidering Esport: Economics and Executive Ownership. Physical Culture and Sport. Studies and Research, 74(1), 43-53.

[9] P Mora and S Héas (2003). Du joueur de jeux vidéo à l'e-sportif: vers un professionnalisme florissant de l'élite? Consommations et sociétés, 2003, 129145.

[10] Christopher A. Paul. 2018. The toxic meritocracy of video games: Why gaming culture is the worst. University of Minnesota Press.

[11] James Phelan. 1989. Reading People, Reading Plots. Character, Progression and the Interpretation of Narrative. University of Chicago Press.

[12] J Phelan, P Rabinowitz, R Warhol, D Herman and B Richardson, 2012 Character, Narrative Theory: Core Concepts and Critical Debates. Ohio State University Press, Columbus. Ohio.

[13] Cornel Sandvoss. 2005. Fans. The Mirror of Consumption. Polity Press, Malden, Massachusetts.
[14] Murray Smith, 1995. Engaging characters. Fiction, emotion, and the cinema. Oxford University Press.

[15] M Smith, 2011. Engaging Characters: Further Reflections, Characters in Fictional Worlds. De Gruyter, New York, USA.

[16] N Taylor, J Jenson and S De Castell (2009). Cheerleaders/booth babes/Halo hoes: pro-gaming, gender and jobs for the boys. Digital Creativity, 20(4), 239-252.

[17] T.L Taylor. 2012. Raising the Stakes. E-Sports and the Professionalization of Computer Gaming. MIT Press, Cambridge, Massachusetts.

[18] R Turtiainen, U Friman and M Ruotsalainen (2018). "Not Only for a Celebration of Competitive Overwatch but Also for National Pride". Sportificating the Overwatch World Cup 2016, Games and Culture, August 2018. DOI: $10.1177 / 1555412018795791$.

[19] G Voorhees, 2015 Neoliberal masculinity: The government of play and masculinity in e-sports, Playing to Win: Sports, Video Games, and the Culture of Play. Indiana University Press, Indiana, USA.

[20] T Välisalo (2017). Engaging with film characters: Empirical study on the reception of characters in The Hobbit films. Fafnir: Nordic Journal of Science Fiction and Fantasy Research, 4 (3-4), 12-30.

[21] H Wirman (2007). "I am not a fan, I just play a lot" - If Power Gamers Aren't Fans, Who Are? Situated Play, Proceedings of DiGRA 2007 Conference.

[22] H Young (2014). Race in online fantasy fandom: Whiteness on Westeros.org. Continuum, 28(5), 737-747. 